\section{FRI0531 DETERMINANTS OF CLINICAL AND RADIOLOGICAL PROGRESSION OF HAND OSTEOARTHRITIS OVER 2 YEARS}

A. Neuprez ${ }^{1}$, A.H. Neuprez ${ }^{1}$, E. Maheu ${ }^{2}$, O. Bruyère ${ }^{1}$, J.-F. Kaux ${ }^{3}$, J.-Y. Reginster ${ }^{1}$ ${ }^{1}$ Department of Public Health, UNIVERSITY OF LIĖGE, Liège, Belgium; ${ }^{2}$ Rheumatology, AP-HP, Saint-Antoine Hôpital, Paris, France; ${ }^{3}$ Rehabilitation and Sports Traumatology Department, University Hospital of Liège, Liège, Belgium

Objectives: The objectives of this prospective observational study were to assess the clinical and radiological changes in hand osteoarthritis (HOA) and to identify the determinants of these changes, over a two year period.

Methods: 203 patients were included in Llège Hand Osteoarthritis Cohort $(\mathrm{LIHOC})$ and followed during 2 years. They met the American College of Rheumatology $x$-ray/clinical criteria for HOA. At baseline, demographic and clinical characteristics of the population were recorded. Various radiological and clinical parameters were selected to investigate progression.

Results: The general health measures remained stables over time. The number of nodes increased significantly over 2 years while the other clinical parameters did not vary significantly over time (number of painful joints at rest or at pressure and swollen joints). The pinch force decreased over time and the grip strength remained stable. The two tools accessing function (FIHOA and AUSCAN), showed a progressive deterioration over time (statistically significant for FIHOA $(p<0.05)$ and borderline $(p=0.17)$ significant for the AUSCAN). Almost all patients showed radiologic change during follow-up. Thus, the radiological scores deteriorated significantly over 2 years. An increase in Verbruggen and KL scores was present in $162(92.04 \%)$ and $174(98.86 \%)$ patients, respectively. 39 patients (22.16\%) had new erosive joints.

From a clinical perspective, using backward logistic regression, diabetes (OR $2.67 \%-95 \% \mathrm{Cl} 1.13-6.33, \mathrm{p}=0.03$ ), high degree of radiologic severity (OR $1.23 \%-95 \% \mathrm{Cl} 1.09-1.39, \mathrm{p}<0.01)$ and age between 40 and 60 (OR $2.67 \%-$ $95 \% \mathrm{Cl} 1.21-5.90, \mathrm{p}=0.02)$ at baseline are predictors of FIHOA worsening overtime. The predictors of AUSCAN progression included the pain intensity (OR $0.98 \%-95 \% \mathrm{Cl} 0.97-0.99, \mathrm{p}<0.01)$ and the degree of radiologic severity (OR $1.06 \%-95 \% \mathrm{Cl} 1.01-1.12, \mathrm{p}=0.03$ ) at baseline.

The following factors are associated with radiological deterioration: symptomatic HOA (OR 2.17\%-95\% Cl 1.04-4.51, $\mathrm{p}=0.04$ ) and the number of severely affected joints at baseline (OR 1.11\%-95\% Cl 1.04-1.18, p<0.01). In contrast, a high number of erosive or remodelled joints (OR $0.89 \%-95 \% \mathrm{Cl} 0.81-0.98, \mathrm{p}=0.02$ ) reduce the risk of radiological disease progression.

Conclusions: These results help to better understand the clinical and radiologic progression of $\mathrm{HOA}$, as well as the determinants that have resulted in them. Disclosure of Interest: None declared

DOI: 10.1136/annrheumdis-2018-eular.4866

\section{FRI0532 \\ RELATIONSHIP BETWEEN PATIENT-REPORTED OUTCOMES AND PROPRIOCEPTIVE ACUITY IN PATIENTS WITH TOTAL KNEE ARTHROPLASTY}

B. Ünver ${ }^{1}$, M. Eymir ${ }^{1}$, V. Karatosun ${ }^{2} .{ }^{1}$ School of Physical Therapy and Rehabilitation; ${ }^{2}$ Department of Orthopaedics and Traumatology, School of Medicine, Dokuz Eylul University, Izmir, Turkey

Background: Total knee arthroplasty (TKA) is offered to patients who have endstage knee osteoarthritis $(\mathrm{OA})$ to reduce pain and improve functional performance. Pain and functional level in patients with TKA can be measured using selfreport questionnaires such as the Numeric Pain Rating Scale (NPRS), Hospital for Special Surgery (HSS), lowa Level of Assistance Scale (ILAS), and lowa Ambulation Velocity Scale (IAVS). Proprioception plays an integral role in neuromotor control of the knee joint and deficits in knee joint proprioception are well documented in individuals with knee osteoarthritis. However, the patient-reported functional level relevance of these deficits is not clear in both individuals with knee $\mathrm{OA}$ and with TKA.

Objectives: The aim of this study was to assess relationship between pre-/postsurgery patient-reported outcomes and proprioceptive acuity in patient with TKA due to knee OA.

Methods: The study group consisted of 68 patients (12 males, 56 females), who underwent primary TKR because of knee arthrosis were included in the study with mean age $64.9 \pm 9.1$ years. Patients were evaluated regarding the knee proprio-

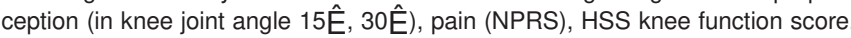
Functional activities were evaluated using the ILAS and walking speed was evaluated using the IAVS. Patients were evaluated preoperatively and at discharge. All patients underwent the same rehabilitation program.

Results: While there were correlations in different rates in individuals with knee OA between patient-reported outcomes and proprioceptive acuity, there were no correlations in individuals with TKA between patient-reported outcomes and proprioceptive acuity. Preoperatively, low-to-moderate significant correlations was found between pain and proprioceptive acuity deficit scores in knee joint angle $15 \hat{E}, 30 \hat{E}(r=-0.196, p=0.046$ and $r=-0.378, p<0.001$, respectively). There were low significant correlations preoperatively between HSS knee score and proprioceptive acuity deficit scores in knee joint angle $15 \hat{\mathrm{E}}, 30 \hat{\mathrm{E}}(r=0.217, p=0.027$, and $r=0.275, p=0.005$, respectively). There were not significant correlations between proprioceptive acuity deficit scores and all other evaluation tests $(p>0.05)$.

Conclusions: There were correlations in different rates in patients with knee OA between patient-reported outcomes and proprioceptive acuity, however there were no correlations in patients with TKA between patient-reported outcomes and proprioceptive acuity. These results suggest that patients who have knee $O A$ and with poor proprioception show more limitation in functional ability and have more pain level. On the other hand, deficits in joint position sense in patients with TKA may be due to factors other than pain and functional disability. Poor proprioception in patients with TKA may be due to surgery related resection of articular cartilage, meniscuses, articular ligaments.

Disclosure of Interest: None declared

DOI: 10.1136/annrheumdis-2018-eular.5869

\section{FRI0533 \\ ASIAN MITOCHONDRIAL DNA HAPLOGROUP B IS ASSOCIATED WITH THE DEVELOPMENT OF KNEE OSTEOARTHRITIS IN KOREAN}

B.S. Koo ${ }^{1}$, Y. Song ${ }^{2}$, S. Lee ${ }^{2}$, Y.-K. Sung ${ }^{3}$, K.-J. Shin ${ }^{4}$, N.H. Cho ${ }^{5}$, J.-B. Jun ${ }^{3}$ ${ }^{1}$ Department of Internal Medicine, Inje University Seoul Paik Hospital, Inje University College of Medicine; ${ }^{2}$ Department of Radiology, ${ }^{3}$ Department of Rheumatology, Hanyang University Hospital for Rheumatic Diseases: ${ }^{4}$ Department of Forensic Medicine, Yonsei University College of Medicine, Seoul; ${ }^{5}$ Department of Preventive Medicine, Ajou University School of medicine, Suwon, Korea, Republic Of

Background: In our previous study, we have conducted a case-control study to demonstrate the mitochondrial DNA (mtDNA) haplogroups in the development of knee osteoarthritis $(\mathrm{OA})$. However, there were no mtDNA haplogroups associated with the development of knee OA.

Objectives: The objective of this study was to elucidate the role of mtDNA hap logroups in the development of knee OA in prospective on-going communitybased cohort.

Methods: This cohort was established in 2001 to investigate the epidemiologic characteristics of major chronic diseases in Korea by the Korean Genome and Epidemiology Study, Centre for Disease Control (KCDC). The epidemiologic data and knee radiographs were obtained from the second follow-up (2005-2006) and the sixth follow-up (2013-2014), and DNA was distributed from the fourth followup (2009-2010). The Kellgren-Lawrence (K/L) score was measured using a knee $\mathrm{X}$-ray taken at each visit. The mtDNA was analysed by multiplex mutagenetically separated polymerase chain reaction to determine the mtDNA haplogroups ( $M$ G, D, D4, D5, M7, M8, M9, M10, N, A, N9, R, F, B). The frequency of the mtDNA haplogroup was compared between the group with knee $O A(K / L \geq 2$ or underwent total knee replacement arthroplasty) and the group without knee $O A(K / L<2)$ at the 6 th follow-up in the cohort of $K / L=0$ at the second follow-up. Multiple logistic regression was used to determine relative risk (RR) of mtDNA haplogroups for OA by adjusting sex, age, and body mass index (BMI).

Results: A total of 1115 epidemiological data, knee radiographs, and DNA samples were distributed. Of these, 572 were cohorts with $K / L=0$ in the second follow up, and 438 underwent knee X-ray examination at the sixth follow-up visit. Among them, 160 were classified as Knee OA by K/L grading and 278 were classified as control group. The mean age $(59.4 \pm 8.5$ and $64.3 \pm 6.8)$, the number of male patients $(61$ [21.9\%] and $11[6.9 \%])$, and the mean BMI $(24.0 \pm 3.1$ and $25.0 \pm 3.0)$ were significantly different between normal and $O A$ group $(p<0.001)$. In comparison of frequency of mtDNA haplogroup between two groups, haplogroup $B$ was significantly higher in $\mathrm{OA}$ group (unadjusted $R R=1.794, p=0.030$ and adjusted $\mathrm{RR}=2.346, \mathrm{p}=0.005$ ).

Conclusions: Our data suggested that mtDNA haplogroup B contributed to the development of knee OA in Korean. Further study is ongoing to confirm the relationship between the progression of knee OA and mtDNA haplogroups.

Acknowledgements: Our previous study was presented at 4th International Congress on Controversies in Rheumatology and Autoimmunity, Bologna, Italy, 9-11 March 2017.

Disclosure of Interest: None declared

DOI: 10.1136/annrheumdis-2018-eular.2999 